\title{
Self-hypnosis for anxiety associated with severe asthma: a case report Ran D Anbar*
}

Address: Department of Pediatrics, State University of New York Upstate Medical University, 750 E. Adams St, Syracuse, NY 13210, United States Email: Ran D Anbar* - Anbarr@mail.upstate.edu

* Corresponding author

Published: 22 July 2003

BMC Pediatrics 2003, 3:7
Received: 06 June 2003

Accepted: 22 July 2003

This article is available from: http://www.biomedcentral.com/|47|-243I/3/7

(C) 2003 Anbar; licensee BioMed Central Ltd. This is an Open Access article: verbatim copying and redistribution of this article are permitted in all media for any purpose, provided this notice is preserved along with the article's original URL.

\begin{abstract}
Background: Management of asthma can be complicated by both medical and psychiatric conditions, such as gastroesophageal reflux, chronic sinusitis, and anxiety. When symptoms of asthma are interpreted without regard to such conditions treatment may yield a suboptimal outcome. For example, anxiety-associated dyspnea, tachypnea, and chest tightness can be mistakenly interpreted as resulting from an exacerbation of asthma. Medical treatment directed only for asthma may thus lead to overuse of asthma medications and increased hospitalizations.
\end{abstract}

Case Presentation: The described case illustrates how a systemic steroid-dependent patient with asthma benefited from receiving care from a pediatric pulmonologist who also was well versed in the diagnosis and treatment of anxiety. By using self-hypnosis, the patient was able to reduce her dependence on bronchodilators. Following modification of her medical therapy under supervision of the pulmonologist, and regular use of hypnosis, the patient ultimately was weaned off her systemic steroid therapy.

Conclusions: This report emphasizes that anxiety must be considered as a comorbid condition in the treatment of asthma. Self-hypnosis can be a useful skill in the treatment of a patient with anxiety and asthma.

\section{Background}

It is well recognized that management of asthma can be complicated by comorbid conditions, such as gastroesophageal reflux and chronic sinusitis. $[1,2]$ When symptoms of asthma are interpreted without regard to such conditions treatment may yield a suboptimal outcome. $[3,4]$ For example, anxiety might not be recognized as a comorbid condition because its associated dyspnea, tachypnea, and chest tightness can be mistakenly interpreted as resulting from an exacerbation of asthma. [5] Medical treatment directed only for asthma in a patient who also has anxiety may lead to overuse of asthma medications and increased hospitalizations. [6] Even when anxiety is recognized, its treatment rarely is undertaken by the health care provider who primarily manages the patient's asthma. Instead, patients sometimes are referred to a mental health professional for management of anxiety. In other circumstances, the anxiety is recognized, but no specific treatment is offered.

The following case report illustrates how a systemic steroid-dependent patient with asthma benefits from receiv- 
ing care from a pediatric pulmonologist who also is well versed in the diagnosis and treatment of anxiety. This example demonstrates a self-hypnosis approach for helping patients with anxiety. This technique can be mastered relatively easily by most health care providers.

\section{Case Presentation}

The patient was diagnosed with asthma at approximately one year of age when she presented with coughing, wheezing, and retractions. Use of p.r.n. albuterol by nebulization relieved these symptoms. At two years of age, she developed a severe exacerbation for which she required admission to the Pediatric Intensive Care Unit. From that time, with the exception of a six month period between the ages of 5 and 6 years, she required systemic steroid therapy to control her symptoms, until her presentation to the Pediatric Pulmonary Center at the age of 12 . During this period, she required 6 admissions to the intensive care unit, and 15 hospitalizations and emergency department visits for asthma exacerbations.

Her mother became aware of the link between the patient's high level of anxiety and asthma exacerbations when the patient was in the Intensive Care Unit at the age of 10. During that hospitalization, the patient became agitated by discomforts such as the temperature of the food, if chest physiotherapy was not performed to her satisfaction, or if she did not receive her therapies "quickly enough." When she was agitated, her muscles tensed, she breathed deeply, cried loudly, and was inconsolable. She could not verbalize her specific concerns but sometimes yelled, "You're not doing anything for me." She was referred for psychological counseling at that time, but after 10 sessions the family felt that the visits were ineffective and discontinued them.

Triggers of the patient's episodes of respiratory distress included decreasing her dose of systemic steroid, viral upper respiratory infections, exposure to cold air, exercise, and strong emotions. She stated that she developed "upper lobe wheezing", with fast and deep breathing when she became frustrated by social situations, such as disagreements with her family or friends. She also stated that these symptoms were different from her "lower lobe wheezing" that she reported as being her "usual" asthma symptom. She added that often she became anxious when her asthma was triggered, and then she would breath deeply and hyperventilate.

Her chronic therapies at the time of presentation to our Center included: Prednisone (15 mg per day), levalbuterol $(0.63 \mathrm{mg}$, by nebulization, four times daily, and p.r.n.); albuterol MDI (two puffs prior to gym); budesonide (three puffs BID); and ipratropium bromide $(0.5 \mathrm{mg}$, by nebulization, p.r.n.) She used her p.r.n. medications approximately every other day for dyspnea.

When she was 5 years old, skin-scratch testing revealed that she was allergic to cats. A sweat chloride test was normal on two occasions. A bronchoscopy and ciliary biopsy were normal. An immunological workup revealed an IgA deficiency, but monthly gamma globulin therapy for nine months, when she was 10 years old, was not associated with an improvement in her symptoms. A 24 -hour $\mathrm{pH}$ probe study, at 11 years of age, revealed only mild gastroesophageal reflux, and therapy with cisapride for five months was not associated with an improvement in her symptoms.

The patient lived with her parents and two brothers in a single-family house. There were no smokers in the home. She was in the seventh grade. She missed "a lot" of school because of her illnesses, and as a result she had some academic difficulties.

Her physical examination revealed a cushingoid appearance, without distress. Her height was $138.1 \mathrm{~cm}$ (at the 3rd percentile for age), and her weight was $53.7 \mathrm{~kg}$ (90th percentile for age). Her respiratory rate was 16 breaths/ min. The lung examination revealed slight expiratory wheezing in the upper airway, which was transmitted to her lower airways. There was good air movement throughout. Otherwise, the examination was unremarkable.

Pulmonary function testing revealed normal expiratory lung flows (forced vital capacity $=119 \%$ of predicted for height and age, forced expiratory volume in one second = $106 \%$, and forced mid-expiratory flow rate $=94 \%$ ). Her inspiratory loop was blunted, consistent with an upper airway problem. Review of her pulmonary function tests from the six years prior to her presentation revealed mostly normal tests. On a few occasions, she had decreased expiratory lung flows, which improved following bronchodilator administration.

The initial assessment at our Center was that the patient had asthma, given the improvement of her symptoms with bronchodilator and systemic steroid therapies. However, it was suspected that an upper airway problem had been playing a role in her presentation, given her report of an "upper airway wheeze" in association with strong emotions, her upper airway expiratory wheezing on physical examination, and the blunting of the inspiratory loop during the pulmonary function testing.

We discussed with the patient that given her severe asthma, it would not be surprising if she became anxious when she developed shortness of breath. The idea was introduced that such anxiety might have led to develop- 
ment of symptoms for which asthma medications might not have been helpful, including vocal cord dysfunction that would account for her upper airway problem. No further testing was performed in order to confirm a diagnosis of vocal cord dysfunction. The patient's anxiety was not assessed formally.

The patient accepted the opportunity to be taught selfhypnosis to control her respiratory symptoms. Instruction was provided over two weeks in two 45-minute sessions by her pediatric pulmonologist who had received training in hypnosis through three 20-hour workshops.

In the first session the patient was taught to hypnotize herself by imagining that she was walking from a beach house towards the waterfront. She imagined herself lying down and relaxing. She was instructed that she could allow her body to relax whenever she touched her index finger to her thumb ("finger relaxation technique"). On the physical examination following hypnosis, her expiratory wheezing had diminished greatly. She was congratulated upon her outstanding hypnotic abilities. She was encouraged to practice hypnosis on a nightly basis, and to apply her "finger relaxation technique" several times a day. Within two weeks of daily utilization of self-hypnosis, she reported that she was able to use hypnosis instead of nebulized levalbuterol approximately half the time.

In the second instruction session the patient was shown how to develop imagery within hypnosis of a "tight airway that opens up" in order to help relieve airway obstruction. She was encouraged to utilize hypnosis as much as she thought was helpful to her. Thereafter, during the patient's follow-up visits for asthma management, the pulmonologist inquired whether she was using hypnosis. The patient was encouraged to continue its use, but was not provided with further instruction regarding how to use hypnosis. The follow-up visits occurred on a monthly basis as the patient's asthma therapy was weaned, and every 2-3 months thereafter.

After three months, she was able to wean herself completely off levalbuterol, which she had been using more than four times a day. She explained that she found the use of hypnosis to be as helpful as levalbuterol in treating her shortness of breath. Further, she reported that her bouts of shortness of breath were much less frequent than prior to the use of hypnosis.

Once her regular use of levalbuterol was discontinued, she began a slow wean off her systemic steroid therapy. Three months into the steroid wean, when she was receiving hydrocortisone (10 mg per day), she required hospitalization for dyspnea that was only partially relieved with the use of nebulized levalbuterol, oral prednisone $(1 \mathrm{mg} / \mathrm{kg} /$ day), and hypnosis. As she appeared very anxious, on the fourth day of the hospitalization, she was prescribed a single dose of clonazepam. She asked to go home on the following day.

Two weeks after this hospitalization her steroid weaning schedule was resumed. She was hospitalized again nine months into the steroid wean, after an exposure to cigarette smoke at a relative's house. At the same time, her mother was in the hospital with complications as a result of a hysterectomy. Two weeks after discharge, her chronic systemic steroid therapy was discontinued. Her medication regimen at the end of the weaning process was: fluticasone ( $110 \mu \mathrm{g}, 2$ puffs twice a day); salmeterol (2 puffs twice a day); theophylline ( $300 \mathrm{mg}, \mathrm{PO}$, twice a day); budesonide nasal spray ( 2 squirts into each nostril per day); and levalbuterol (0.63 mg, by nebulization, p.r.n.), which she utilized once a month in treatment of shortness of breath.

She was well for the subsequent year with the exception of three asthma exacerbations induced by upper respiratory infections, for which she required short courses of oral prednisone. Over the year her height increased to $150 \mathrm{~cm}$ (up to the 10th percentile for age), while her weight increased to $66.7 \mathrm{~kg}$ (thereby remaining at the 90th percentile). She continued to use self-hypnosis on an almost daily basis to decrease a sensation of dyspnea, relax, reduce her frustrations, fall asleep, and improve her school performance.

\section{Discussion}

Indicators in this patient's presentation that she might respond to use of hypnosis include her lack of complete response to medical therapy, report of dyspnea despite normal lung function, [7] symptoms and signs suggestive of upper airway involvement, and report that strong emotions and anxiety triggered some of her respiratory symptoms.

The patient wanted to learn how to use hypnosis to control her anxiety. [8] She improved greatly following selfhypnosis instruction, as she was weaned off chronic systemic steroid therapy and frequent daily use of a nebulized bronchodilator. Thus, this example emphasizes that anxiety must be considered as a comorbid condition in the treatment of asthma.

Children's report of anxiety has been shown not to be correlated with the severity of their asthma. [9] Thus, the possibility of anxiety as a cause of respiratory symptoms should not be restricted only to patients with severe asthma. Further, as symptoms of anxiety (e.g., dyspnea and chest tightness) may be difficult to differentiate from those of asthma, self-hypnosis might be offered as therapy 
for any interested patient with asthma who does not respond easily to medical therapy. While formal assessment of anxiety is not necessary prior to introduction of self-hypnosis therapy, interested clinicians might utilize anxiety inventories with both patients and their parents in order to help assess the efficacy of therapy. $[9,10]$

While some anxiety is useful in motivating patients to avoid asthma triggers, and rapidly assess and respond to respiratory symptoms, excessive anxiety has been shown to adversely affect management of asthma. [5] For example, patients who hyperventilate during a panic attack, can develop increased bronchospasm as a result of increased inhalation of cold and dry air. [6] Also, patients who have a high level of anxiety tend to perceive their degree of airway obstruction inaccurately, which leads to inappropriate use of asthma medications as well as health care resources. [5]

The anxiety reduction achieved with hypnosis by the patient may have promoted an improvement in her subjective assessment of her respiratory status. [11] Such an improvement can allow patients to reduce their perception of dyspnea and thereby decrease their reliance on bronchodilator therapy.

Another potential benefit of hypnosis facilitated stress reduction for patients with asthma is its effect on the body's immune function. [12] For example, psychological stress has been shown to cause a shift in the cytokine type1/type-2 balance toward a predominant type- 2 response that is characteristic of asthma. [13] It remains to be studied whether such a shift and other stress induced changes in the immune system predispose patients to developing or worsening asthma, or if anxiety reduction alters the immune response sufficiently to decrease patients' asthma risk.

In addition to instruction in self-hypnosis or other relaxation techniques, [14] the anxiety of patients with asthma can be improved by providing them with information regarding the prognosis of their disease, as well as training in accurate assessment of their triggers and degree of airway obstruction. [5] Thus, the patient in this report also may have improved as a result of her close medical supervision.

The patient's improvement may in part have been the result of the efficacy of hypnosis in treating vocal cord dysfunction. [15] However, a definitive diagnosis of vocal cord dysfunction was not made in this case, as the patient did not undergo a laryngoscopic examination of her vocal cords while she was symptomatic. [16] Nevertheless, as may have occurred in this case, self-hypnosis has been shown to help resolve symptoms suggestive of vocal cord dysfunction even in the absence of a definitive diagnosis. [7]

The ability of a single care provider to manage both the patient's medical and psychological needs appeared to facilitate her therapy. However, some medical providers may not have the background to manage anxiety without collaboration with an individual trained in psychology or psychiatry.

Clinicians interested in learning how to use hypnosis within their medical practice should enroll in training workshops, as hypnosis is learned best through exposure to a variety of educational experiences, including didactic presentations, demonstrations, modeling by different hypnotherapists, and small group practice sessions. [17] Such workshops are administered by the American Society of Clinical Hypnosis, the Society for Clinical and Experimental Hypnosis, and the Society for Developmental and Behavioral Pediatrics. Clinicians can expect that following participation in one or two 20-hour workshops that they will have sufficient knowledge of hypnosis to be able to apply it in several clinical situations.

\section{Conclusions}

Anxiety must be considered as a comorbid condition in the treatment of asthma. Treatment of anxiety with selfhypnosis or other measures is essential for the improvement in the symptoms of some patients.

\section{Competing interests}

None.

\section{Author's contribution}

The author is the pediatric pulmonologist described in this article. He wrote the manuscript.

\section{Acknowledgements}

Written consent was obtained from the patient and her mother for publication of this study.

\section{References}

I. Bresciani $M$, Paradis $L$ and Des Roches $A$ et al.: Rhinosinusitis in severe asthma J Allergy Clin Immunol 2001, 1 07:73-80.

2. Field SK and Sutherland LR: Does medical antireflux therapy improve asthma in asthmatics with gastroesophageal reflux? A critical review of the literature Chest 1998, I | 4:275-283.

3. Rachelefsky GS, Katz RM and Siegel SC: Chronic sinus disease with associated reactive airways disease in children Pediatrics 1984, 73:526-529.

4. Harding SM, Richter JE and Guzzo MR et al.: Asthma and gastroesophageal reflux: acid suppressive therapy improves asthma outcome Am J Med 1996, I00:395-405.

5. ten Thoren $C$ and Petermann E: Reviewing asthma and anxiety Respir Med 2000, 94:409-4I5.

6. Carr RE: Panic disorder and asthma J Asthma 1999, 36:|43-I52.

7. Anbar RD: Self-hypnosis for management of chronic dyspnea in pediatric patients Pediatrics 200I, 107: [http://www.pediatrics.org/cgi/content/full//07/2/e2I].

8. Lynn SJ, Kirsch I, Barabasz A, Cardena E and Patterson D: Hypnosis as an empirically supported clinical intervention: the state of 
the evidence and a look to the future Int J Clin Exper Hypnosis 2000, 48:239-259.

9. Wamboldt MZ, Fritz G, Mansell A, McQuaid El and Klein RB: Relationship of asthma severity and psychological problems in children J Am Acad Child Adolesc Psychiatry 1998, 37:943-950.

10. Butz AM and Alexander C: Anxiety in children with asthma J Asthma 1993, 30:199-209.

II. Hackman RM, Stern JS and Gershwin ME: Hypnosis and asthma: A critical review J Asthma 2000, 37:I-I5.

12. Gruzelier $\mathrm{JH}$ : A review of the impact of hypnosis, relaxation, guided imagery and individual differences on aspects of immunity and health Stress 2002, 5: 147-163.

13. Marshall GD Jr and Agarwal SK: Stress, immune regulation, and immunity: applications for asthma Allergy \& Asthma Proceedings 2000, 2 I:24I-246.

14. Huntley A, White AR and Ernst E: Relaxation therapy for asthma: a systematic review Thorax 2002, 57:|27-|3|

15. Anbar RD: Hypnosis in pediatrics: applications at a pediatric pulmonary center BMC Pediatr 2002, 2: I I [http://www.biomedcentral.com/|47I-243I/2/II]

16. Anbar RD and Hehir DA: Hypnosis as a diagnostic modality for vocal cord dysfunction Pediatrics 2000, 106: [http://www.pediatrics.org/cgi/content/full/|06/6/e8I].

17. Wark DM and Kohen DP: A spiral curriculum for hypnosis training Am J Clin Hypn 2002, 45:1 19-128.

\section{Pre-publication history}

The pre-publication history for this paper can be accessed here:

http://www.biomedcentral.com/1471-2431/3/7/prepub

Publish with Bio Med Central and every scientist can read your work free of charge

"BioMed Central will be the most significant development for disseminating the results of biomedical research in our lifetime. "

Sir Paul Nurse, Cancer Research UK

Your research papers will be:

- available free of charge to the entire biomedical community

- peer reviewed and published immediately upon acceptance

- cited in PubMed and archived on PubMed Central

- yours - you keep the copyright

Submit your manuscript here:

http://www.biomedcentral.com/info/publishing_adv.asp 